\title{
Istanbul in den Wunderzeichenberichten des 16. Jahrhunderts
}

\author{
16. Yüzyıl Almanca El İlanlarında İstanbul
}

\section{Leyla COŞAN*}

Zusammenfassung: Seit dem antiken Zeitalter gibt es Wunderzeichenberichte. Insbesondere mit der Eroberung Konstantinopels und der sich europaweit verbreitenden “Türkenfurcht”, nahm auch die Stadt "Istanbul” ihren Platz in diesen Wunderzeichenberichten ein. Kometen und andere Himmelszeichen, Naturkatastrophen, Missgeburten etc. wurden auch im Zusammenhang mit der Türkenfurcht als besondere Zeichen gesehen, durch die auf ein in der Zukunft liegendes Ereignis gedeutet werden sollte. Auf der einen Seite führte die Furcht vor dem Feind dazu, dass die Bevölkerung Trost und Hoffnung in diesen Wunderzeichenberichten zu finden glaubte. Auf der anderen Seite wurden diese Berichte auch gezielt als Propagandamittel eingesetzt, um die Bevölkerung zur Gegenwehr zu ermutigen. In diesem Zusammenhang sollen anhand exemplarischer Flugschriften des 16. Jahrhunderts Wunderzeichenberichte zur Analyse herangezogen werden, in denen die Stadt “Istanbul” als Hort des Bösen, bzw. als Sitzstätte des Feindes thematisiert wird.

Schlüsselwörter: 16. Jahrhundert, Istanbul, Flugschriften, Wunderzeichen, Türkenfurcht

Öz: Antikçağdan bu yana mucizevi işaret haberleri yazılmaktadır. Özellikle de İstanbul'un fethi ve Avrupa geneline yayılan Türk korkusu dolayısıyla, İstanbul şehrinin mucizevi işaret haberlerinde yerini almasına neden olmuştur. Kuyruklu yıldızlar ve diğer gökyüzü kaynaklı işaretler, doğal afetler, doğumsal anomaliler vd. Türk korkusu ile ilişkilendirilmiş ve gelecekteki olayların yorumlanmasına yol açan özel işaretler olarak kabul edilmiştir. Bir yandan mevcut Türk korkusu nedeniyle toplum bu tür mucizevi işaret haberlerinde teselli ve ümit bulacağına inanmakta, diğer yandan bu tür haberler propaganda aracı olarak kullanılarak toplumu savunma ve savaş için cesaretlendirmeye çalışmaktadır. Bu bağlamda kötülüğün kaynağı ve düşmanın yurdu olarak kabul edilen İstanbul şehrinin yeri, önemi ve fonksiyonları, 16. yüzyıl mucizevi işaret haberleri örneğinde incelenecektir.

Anahtar sözcükler: 16. Yüzyıl, İstanbul, El İlanları, Mucizevi İşaretler, Türk Korkusu

\section{Über die Bedeutung der Stadt Istanbul in der Geschichte}

Istanbul ist die Stadt, die das Erbe zweier großer Imperien, des Byzantinischem und des Osmanischen, beheimatet. Sie wurde unzählige Male von verschiedenen Völkern, wie den Arabern, Persern, Awaren, Bulgaren und Russen belagert, aber der Sage und Überlieferung nach sollte ein türkischer Sultan die Stadt erobern. Nach der arabisch-türkischen Legendentradition soll der Prophet Muhammed gesagt haben: "Ihr werdet Qostantinija erobern. Heil dem Fürsten und dem Heere, denen solches beschieden” (Kocadoru 1990, 77-78). Eine Miniatur aus dem "Mi'rağnama"-Blatt (Himmelreise Mohammeds) aus einem Täbris Saray-Album, Anfang des 14. Jahrhunderts veranschaulicht diese Legende (Ipşiroğlu 1980). Darauf ist zu sehen, wie der

\footnotetext{
*Prof. Dr., Marmara Üniv., Fen-Edebiyat Fak., Alman Dili ve Edebiyatı Bölümü, İstanbul. leylacosan@marmara.edu.tr
} 
Engel Gabriel dem Propheten Muhammed ein Stadtbild überreicht, auf dem vermutlich Konstantinopel abgebildet ist. "...Auf der oberen Hälfte des Bildes sitzt Mohammed in geflammter Mandorla auf einem mit geometrischen Motiven gemusterten seldschukischen Teppich. Die erhobene rechte Hand (Redegeste) weist darauf hin, daß er spricht. Er wendet sich an zwei Figuren, die ihm gegenüber in ehrfurchtvoller Haltung kniend hocken. Über diese Figuren schwebt eine gekrönte Engelsgestalt mit ausgebreiteten Flügeln und überreicht Mohammed ein Stadtbild, das durch einen Wasserlauf in zwei Teile getrennt ist. Die Annahme, da $\beta$ mit dieser Darstellung die spätere islamische Metropole am Goldenen Horn gemeint sein kann, wird durch den blauen Wasserweg im Bild und durch die Osmanischen Bauformen der Moschee bekräftigt...” Die Osmanen zeigten während des gesamten 14. Jahrhunderts Interesse an Byzans und Istanbul. Als erste starke Belagerung ist jedoch der Versuch von Sultan Yıldırım Bayezid I. (1360-1403) zu nennen, der indem er die Stadt im Jahre 1395 belagerte, diese Prophezeiung verwirklichen wollte. Auch der Sohn Yıldırım Beyazids, Musa Çelebi (?-1413), unternahm im Jahre 1411 einen Belagerungsversuch, der jedoch ebenfalls erfolglos blieb. Während der Epoche von Sultan Murad II. (1404-1451), im Jahre 1422 wurde die letzte große Belagerung, die vor der Eroberung Istanbuls stattfand, verwirklicht. Diese Bestrebungungen wurden von seinem Sohn Mehmet II. (1432-1481) fortgeführt. Im Jahre 1453 wurde die seit eh und je begehrte Stadt von ihm belagert und in kurzer Zeit erobert.

Aus osmanischer Sicht war die Eroberung Konstantinopels nicht nur ein Ergebnis der politisch-wirtschaftlichen Expansionsbestrebungen der osmanischen Herrscher, sondern auch eine religiös motivierte Handlung, die in Mehmet dem Eroberer vollendet und verwirklicht wurde. Dieses Ereignis prägte die christliche Welt für lange Zeit, vor allem auch deshalb, weil die Bedrohung Mitteleuropas durch die Türken anhielt. Aus diesem Grunde schien jedes außerordentliche Naturereignis auf die Machenschaften des Feindes hinzudeuten, stellt Pierre Simon de Laplace anhand des Kometen von 1456 fest: "So verbreitete der lange Schweif des Kometen von 1456 großen Schrecken in Europa, wo man schon über die schnellen Fortschritte der Türken bestürzt war, die kurz zuvor das abendländische Kaiserthum zerstört hatten, und der Papst Callixtus verordnete öffentliche Gebete, in denen man den Komet und die Türken verfluchte" (Langsdorf 1819, 5).

Seit dem antiken Zeitalter gibt es Wunderzeichenberichte. Vor allem aber in der frühen Neuzeit treten sie in grosser Zahl in Flugblättern und Flugschriften auf. Himmelszeichen, Naturkatastrophen, Missgeburten etc. wurden als besondere Zeichen gesehen, durch die auf ein in der Zukunft liegendes Ereignis gedeutet werden sollte. Insbesondere mit der Eroberung Konstantinopels und der sich europaweit verbreitenden "Türkenfurcht", nahm auch die Stadt "Istanbul" und der "Türke" seinen Platz in diesen Wunderzeichenberichten ein. Auf der einen Seite führte die Furcht vor dem Feind dazu, dass die Bevölkerung Trost und Hoffnung in diesen Wunderzeichenberichten zu finden glaubte. Auf der anderen Seite wurden diese Berichte auch gezielt als Propagandamittel eingesetzt, um die Bevölkerung zur Gegenwehr zu ermutigen. Die Anzahl der Flugblätter und Flugschriften, in denen man sich mit der von den Türken ausgehenden Gefahr m.a.W. dem Türkenbild auseinandersetzt, ist nicht gering zu schätzen. Demgegenüber stellen die Wunderzeichenberichte, in denen die Stadt Konstantinopel bzw. Istanbul thematisiert wird, eher eine Seltenheit dar. Aus diesem Grunde ist es Ziel dieses Beitrags festzustellen, in welchen Zusammenhängen die Stadt Istanbul in Erscheinung tritt. Diesbezüglich sollen anhand der Flugblätter und -schriften des 16. Jahrhunderts Wunderzeichenberichte zur Analyse herangezogen werden, in denen die Stadt "Istanbul" aus der Sicht des Gegners thematisiert wird. Neben dem Kometen als Himmelserscheinung, wurden auch Naturkatastrophen wie beispielsweise Erdbeben, Wolfsangriffe und Heuschreckenplagen 
verzeichnet. Zuletzt soll auf die Frage eingegangen werden, ob diese in Verbindung mit der Stadt Istanbul beschriebenen Erscheinungen im Bereich des real Möglichen liegen oder dem Bereich des Phantastischen bzw. des Irrealen zuzurechnen sind. Zuvor sollen aber kurz die Begriffe Flugblatt und Flugschrift erläutert werden.

Die Erfindung des Buchdrucks ermöglicht es ab Mitte des 15. Jahrhunderts erstmals Texte in hoher Auflagenzahl zu günstigen Preisen zu produzieren. Unter diesen Bedingungen entstehen auch die sogenannten Flugblätter und Flugschriften [Die Begriffe Flugblatt und Flugschrift sind nicht zeitgenössisch, sondern wurden erst 1787 bzw. 1788 von Christian Daniel Schubart "aus dem frz. feuille volante gewonnen". In der frühen Neuzeit selbst wurden die beiden Druckmedien in Messkatalogen und Druckeranzeigen unter die Druckerzeugnisse buoch oder buechlin subsumiert. Die häufigste Bezeichnung lautet jedoch Neue Zeitung (Schwegler 2002, 20)] als "selbständige, nichtperiodische und nicht gebundene Druckschriften" (Köhler 1987, 57). Sie werden vorwiegend auf Marktplätzen, Kirchen oder in Wirtshäusern von Händlern oder Kolporteuren verkauft. Anders benannt werden diese Druckerzeugnisse auch als Neue Zeitung, was zunächst nicht viel mehr bedeutet als neue Nachricht bzw. aktuelle Neuigkeit. Der vermutlich zum erstenmal 1502 auftauchende Begriff wird bereits im Jahr 1567 zur Amtssache und zwar "als Bezeichnung für die reinen Nachrichtenblätter, die als die eigentlichen Vorläufer der späteren periodischen Zeitungen anzusehen sind" (Lindemann 1969, 65). Diese Zuschreibung impliziert damit bereits deren Zweck und Funktion. Neue Zeitung als Hyperonym für Flugblatt und Flugschrift und seine Übertragung ins Neuhochdeutsche mit aktuelle Nachricht zeigt ebenfalls, dass hier mehr Bezug auf den Inhalt als auf die äußere Form genommen wird. Da der Begriff auch in Wunderzeichenberichten auftaucht, ist nach Schwegler weiterhin anzunehmen, "dass der Begriff Nachricht dabei sehr weit gefasst werden muss, also nicht nur ,objektive Information" meint, sondern auch Sensationsmeldungen miteinschließt" (Schwegler 2002, 20-21).

Die Illustration ist eines der wichtigsten Unterscheidungskriterien zwischen den einblättrigen Flugblättern und den mehrseitigen Flugschriften. Doch welches Kriterium lässt die Flugschriften von anderen heftchenartigen Kleinschriften unterscheiden? "Jede Flugschrift über wunderbare Erscheinungen, besteht aus einem Berichtteil und einem Deutungsteil” (Schwegler 2002, 18). Dies scheint nach Schwegler vor allem für Wunderzeichen-Flugschriften ein zentrales Merkmal zu sein (Schwegler 2002, 18). Auch sprachliche Besonderheiten lassen sich an Wunderzeichen-Flugschriften erkennen. Bestimmte Schlagwörter auf den Titelblättern, wie beispielsweise Newe Zeitung, Wahrhaftiger Bericht, Erschröckliche Geschicht (Schwegler 2002, 18-19) gelten als wichtiges Unterscheidungsmerkmal. Aber auch der sich an eine breite Öffentlichkeit richtende und meist auf Deutsch abgefasste Text und die meist genaue Datierung und Lokalisierung des Geschehens sind als wichtige Unterscheidungskriterien anzusehen.

Obwohl Wunderzeichenberichte in diesen Textgattungen vor allem in der frühen Neuzeit in hoher Zahl auftreten, sind die in ihnen behandelten Themen, wie bereits erwähnt, nicht neu. Diese "außernatürlichen Begebenheiten in der Natur" (Zedler 1749, Bd. 59, Sp. 2149), Wunderzeichen bzw. lat. Prodigia [Mit Wunderzeichen bzw. Prodigia meinte man die "Andeutungen der Zukunft, die in ihrem Erscheinen wunderbar, d.h. unerklärlich nach dem gewöhnlichen Gange der Natur sind, und deren Deutung man von gewissen Menschen erwartet" (zuletzt am 08.01.2011, unter http://kruenitz1.uni-trier.de/cgi-bin/getKRLemmasel.tcl?)] genannt, wurden schon in der Antike als besondere Zeichen gedeutet (Rosenberger 1998, 91-92). Bei dem Kompositum Wunder-Zeichen handelt es sich damit einmal um das Ereignis selbst, welches als Wunder verstanden wird und von daher als nicht auf natürlichem Wege erklärbar gilt. Zum Anderen habe dieses Wunder einen zeichenhaften Charakter, wie Schwegler feststellt, und "geschieht also nicht um seiner selbst willen, sondern um etwas (Höheres) anzuzeigen" (Schwegler 2002, 32). Das kann sowohl ein zukünftiges Ereignis, als auch den Zorn der Götter 
meinen. Diese unterschiedlichen Ausprägungen des zeichenhaften Charakters müssen deshalb immer vor dem jeweiligen historischen Kontext betrachtet werden (Schwegler 2002, 32).

Dass das Interesse für Wunderzeichen bzw. Prodigien im 16. Jahrhundert stieg, ist nach Rudolf Schenda hauptsächlich mit drei Faktoren in Verbindung zu bringen: "der Sensationshunger des Menschen", die "Kompillationsmanier der Humanisten" und "der Gedanke, dass sowohl die religiösen und politischen Wirren als auch die sozialen Missstände auf dem Zorn Gottes beruhen, der täglich neue Zeichen geschehen lässt, um die Menschen zur Buße zu mahnen und an das nahende Weltende zu erinnern" (Schenda 1963, Sp. 640f.).

Die Stadt Istanbul hingegen wird in den Wunderzeichenberichten im Zusammenhang mit Himmelszeichen, Naturkatastrophen und seltener mit zoologischen Missgeburten thematisiert.

\section{Himmelszeichen}

Mehr als die Hälfte der Flugblätter mit Wunderzeichenberichten setzen sich mit bizarren Himmelsphänomenen auseinander. Naturwissenschaftliche Studien belegen allerdings, dass in der Realität, die Anzahl der verzeichneten Himmelserscheinungen um einiges geringer ist. Der Grund für die Vielzahl der Wunderberichte liegt deshalb darin, das sehr häufig zu einer Erscheinung verschiedene Flugblätter und Flugschriften ausgegeben worden sind.

Wunderflugblätter über Himmelszeichen lassen sich in zwei Gruppen unterteilen: Astronomische und meteorologische Erscheinungen. In den meisten Fällen ist jedoch festzustellen, dass beide Phänomene zugleich thematisiert werden, und ineinander übergreifen. Der Astronomie zuzurechnen sind Kometen, Sternschnuppen, Meteore, Sonnen- und Mondfinsternisse. Darunter nimmt das Erscheinungsbild eines Kometen in den Flugblättern der Neuzeit den ersten Rang ein und wurde nahezu fast immer als unheilvolles Vorzeichen gedeutet. Trotz allem gab es auch, wenn auch nur vereinzelt, positive Deutungen der Kometen, wie z.B. Johannes Capistrano (1386-1456), der den Kometen als gutes Zeichen deutete und davon ausging, dass er den Sieg des Christentums gegenüber den Türken anzeige (Vgl. Heitzer 1995, 48; Coşan 2010, 169-180). Haloerscheinungen (Nebensonnen und Lichtringe), Nordlichter, seltsame Wolkenbilder, Dämmerungserscheinungen etc. sind den meteorologischen Erscheinungen zuzurechnen. Diese sind entweder vollkommen phantastischer Natur oder unterliegen zumindest einer solch phantastischen Ausdeutung. Ihr symbolischer Gehalt zieht die Bevölkerung in ihren Bann, denn die Menschen sehen bei diesen Erscheinungen weit über das eigentliche Naturphänomen hinaus und deuten diese mit Bildern sich gegenseitig bekämpfender Heere oder christlicher Symbole, wie bspw. die Rute als drohende Strafe Gottes, aus.

\section{Naturkatastrophen}

Unter den zahlreichen Naturkatastrophen spielt das Erdbeben in Zusammenhang mit den Türkenflugblättern und -schriften eine wichtige Rolle, da es auch am häufigsten thematisiert wird. Seit dem antiken Zeitalter gilt das Erdbeben als wichtiges Wunderzeichen, denn Erdbeben gehören zu den Zeichen, die nicht nur Strafen ankündigen, sondern diese auch mit sich bringen. Da Erdbebenkatastrophen auch in der Neuzeit nicht vollständig zu erklären sind, obliegen sie zudem einer Ausdeutung als Unheilsbringer. Wird die Bevölkerung nicht selbst heimgesucht, sondern vernimmt nur den Schicksalsschlag aus den Berichten Anderer, so versteht sich dies als Warnung vor ähnlichen Strafen. Trifft einen das Unheil jedoch selbst, so habe man sich durch eigene Versündigung unmissverständlich den Zorn Gottes zugezogen (Vermij 2003, 235-254; Sallmann 2003, 157-178). 
Anhand zwei repräsentativer Wunderzeichenberichte, einem Flugblatt und einer Flugschrift, soll im Folgenden auf die Bedeutung von Naturkatastrophen und Himmelzeichen in Istanbul verwiesen werden. Zudem soll festgestellt werden, ob diese außerordentlichen Erscheinungen der Realität entsprechen oder als Produkt der Phantasie anzusehen sind.

\section{Flugblatt: Naturkatastrophen und Himmelzeichen in Istanbul als Wunderzeichen um 1556}

Im Jahre 1556 erscheint in Nürnberg der anonyme Einblattdruck "Ein erschröcklich wunderzeichen / von zweyen Erdbidemen / welche geschehen seind zu Rossanna unnd Constantinopel / Im M.D.LVI. Jar" (Heitzer 1995, 262). Das Flugblatt enthält eine Abbildung, auf der die vom Erdbeben erschütterte Stadt Istanbul zu sehen ist (S. auch Bischoff 1986, 87; Heß 1911, 7; Heitzer 1995, Abb.61). Die mit emporgehobenen Armen, hin und her rennend hilfesuchenden Menschen und zahlreiche eingestürzte Gebäude sollen auf die Stärke des Erdbebens verweisen. Desweiteren ist auf der Abbildung ein Komet mit außerordentlich langem Schweif, ein Halbmond und ein hell leuchtender Stern zu sehen. Laut angeblicher Augenzeugen, die die Glaubwürdigkeit des angeblich Gesehenen bezeugen sollen, wird neben einem Kometen, welcher zwölf Tage lang am Himmel zu sehen gewesen sei auch von einem schrecklichen Erdbeben berichtet, welches in der Stadt erheblichen Schaden angerichtet haben soll. Demzufolge seien viele Türme sowie unzählige Gebäude eingestürzt und viele Menschen ums Leben gekommen. "Auch der herrliche Tempel St. Sophie (welchen gemeinicklich die türkischen Keyzer pflegten zu besuchen) hat durch solchen erdbidem grossen Schaden empfangen”. Das Erdbeben soll drei Tage angedauert haben, wobei am letzten ein außerordentlich hell leuchtender Stern in unmittelbarer Nähe des Mondes erschienen sei.

Diese Wunderzeichen verweisen auf die biblische Verheißung des Jüngsten Tages, also auf das bevorstehende Weltende. Sie haben demnach eine mahnende Funktion und rufen die Menschen zur Buße auf. Durch die hier dargestellte Zerstörung Konstantinopels wird der türkische und, durch die Verwüstung des so benannten Sophientempels (Hagia Sophia), der römische Antichrist bestraft.

Interessant dabei ist, dass es im Jahre 1556 in Istanbul tatsächlich ein Erdbeben gegeben hat. Laut Orhan Sakin wurde Istanbul am 11. Mai 1556 am frühen Morgen von einem "ziemlich großen" Erdbeben erschüttert. "Zahlreiche Häuser sollen dabei zerstört worden sein” [Tevârih-i Âl-i Osman, Topkapı Sarayı Kütüphanesi Revan Kitapları No: 1100, vr. 105b, zit. nach Cezar 1963, 384. Cezar datiert aber das Erdbeben auf den 30. April 1557. Sakin, der sich ebenfalls auf die selben Quellen beruft, datiert das Erdbeben auf den 11. Mai 1556 (2002, 68) Auch Ambraseys \& Finkel setzen sich mit den Nachrichten auseinander, die zu diesem Thema erschienen sind (2006, 439)]. Die am Goldenen Horn gelegenen Mauern und ein Teil der Mauern des Topkap1 Palastes haben erheblichen Schaden abbekommen (Sakin 2002, 68).

\section{Flugschrift: Naturkatastrophen, Himmelszeichen und Fabelwesen in Istanbul um 1542}

Anhand einer repräsentativen Flugschrift aus dem Jahre 1542 soll auf die Rolle Istanbuls verwiesen werden. Dabei soll auch darauf eingegangen und festgestellt werden, inwiefern die dargestellten Ereignisse der Realität entsprechen könnten.

Diese Flugschrift, die unter dem Titel Newe Zeytung von Constantinopoli erscheint, thematisiert die bizarren Ereignisse in Istanbul, Edirne und Gelibolu. Der Nachrichtenmeldung zufolge wurden dort täglich außergewöhnliche Erscheinungen gesichtet, die zugleich auch als Wunderzeichen gedeutet wurden. Neben dem Kometen als Himmelserscheinung, wurden auch Naturkatastrophen wie beispielsweise Erdbeben, Wolfsangriffe und Heuschreckenplagen verzeichnet. 
Im Untertitel hingegen wird darüber berichtet, dass über dem türkischen Palast vierzig Tage lang ein Komet und ein feuerspeiender Drache gesehen wurde. Der Drache hingegen soll den neuen Palast und die Schätze des türkischen Kaisers in Brand gesteckt und vernichtet haben. Außerdem werden über die verheerenden Folgen von Donner, Sturm, Hagel und Regen berichtet, sowie die von großen Erdbeben ausgehenden katastrophalen Schäden auf die türkischen Städte "Constantinopoli, Adrinopoli und Callipoli" [Newe zeytung von Constantinopoli. Von eynem Comet/der biß in die 40. tag am hymel ober des Türcken pallast gestanden ist. Von einem fewren Trachen der dem Türcken seinen schatz vnd das new Schloß verprent vnd verderbt hat. Von tonnern/ winden/ hagel vnd schaur/ vnd was schaden sie gethan haben. Von grossen Erdtbidmen. Was zu Constantinopoli Andrinopoli vnd Callipoli geschehen ist. Von den grausamen sachen vn Rumorn von Kriegs geschrey vnd von der grossen suma Wölffen/ so 3. tag in der stat Constantinopoli/ was schaden sie gethan haben. Die auslegung aller obgemelter geschehenen Sachen/Ist geschehen durch zwölff des Türckischen Keysers fürnembste Astronomi/ und sich wie Christen erzeigt haben/ darüb sie der groß Türck hat wöllen verprennen lassen/ un wie sie wunderbarlich bei dem Leben erhalten sein worden/ und wie sie der groß Türck zum heiligen grab verordnet hab. Von der grossen suma Hewschrecken/ die alles das in weytem feld biß in die 20.Welschmeyl verderbt haben 1542 (BSB). Newe Zeitung von Constantinopoli ... von eynem Comet S.l. 1542 [VD16 N 945] Persistente Identifier (Werk): urn:nbn:de:bvb:12-bsb00025126-7 Persistente Identifier (Seite): http://daten.digitalesammlungen.de/ db/0002/bsb00025126/image_1]. Der Untertitel des Wunderzeichenberichtes enthält nicht nur die Information, dass furchterregender Kriegsgeschrei vernommenen wurde, sondern zudem auch eine große Summe von Wölfen drei Tage lang die Stadt Constantinopoli belagert und großen Schaden angerichtet hätten. Weiterhin wird erwähnt, dass diese vermeintlichen Ereignisse von zwölf christlichen Astrologen des türkischen Kaisers gedeutet wurden, woraufhin dieser die Astrologen auf dem Scheiterhaufen verbrennen lassen wollte. Diese aber entkamen auf wundersame Weise dem Tod, was den Kaiser dazu veranlasste ihnen eine Wallfahrt zu gewähren. Zuletzt wird im Untertitel auf eine Heuschreckenplage verwiesen, die auf den Feldern ebenfalls erheblichen Schaden angerichtet hat (Newe zeytung von Constantinopoli).

Nach diesem äußerst detaillierten Titel und Untertitel werden alle Ereignisse sehr präzise dargestellt. Auffallend und kennzeichnend für den Wunderzeichenbericht ist, dass die Informationen über die Ereignisse in Istanbul von einer Person stammen, der unmittelbarer Zeuge der sonderbaren Geschehnisse war. Diesem unmittelbaren Zeugenbericht zufolge soll Istanbul nicht nur vierzig Tage lang vom Erdbeben erschüttert, sondern zugleich auch etliche durch ihre Größe auffallende Kometen am Himmel gesichtet worden sein, woraufhin sich "der gro $\beta$ Türck gen Calliopoli gethan hat" (Newe zeytung von Constantinopoli).

Am Freitag, dem 9. Juni soll dem Autor zufolge über dem "schlo $\beta$ des Türcken zu Constantinopoli, eyn mercklicher grosser grausamer Trach", der Feuer speit, am Himmel gesehen worden sein. Dieser soll das neue Schloss des Kaisers samt aller Schätze in Brand gesteckt haben. Das nicht zu löschende Feuer führte dazu, dass am Ende zwei Drittel des Schlosses in Schutt und Asche lag. Die Ereignisse um den feuerspeienden Drachen erinnern an den Brand des Palastes in Istanbul um das Jahr 1540. Wenn man vor Augen hält, dass in der frühen Neuzeit die Daten oft verändert oder nicht so genau genommen worden sind, dann ist es durchaus möglich, das die realen Ereignisse um diese Feuerkatastrophe dem Autor zugleich auch als Inspiration für seine eigene Schrift gedient haben könnte. Mustafa Cezar, der sich mit Bränden und Naturkatastrophen in Istanbul zur Zeit der Osmanen auseinandergesetzt hat, hebt hervor, dass am 5. Februar 1540, während der Abwesenheit des Herrschers, ein verheerender 
Brand das alte Schloss vernichtete (Cezar 1999, 690). Der Nachrichtenmeldung zufolge handelt es sich allerdings um das "neue" Schloss. Dieser Unterschied beruht vermutlich auf einem Informationsfehler oder aber auf der Tatsache, das der christliche Leser wahrscheinlich eine größere Genugtuung empfand, wenn es sich um das "neue" Schloss des osmanischen Herrschers, also des zu diesem Zeitpunkt von der christlichen Welt gefürchteten Feindes handelte.

Am zehnten Tag wüteten Blitz und Donner über der Stadt. Auch der Palast des türkischen Herrschers blieb nicht verschont, so dass seine Lieblingsharemsdame, genannt "die schöne Brandonia", vom Blitz getroffen starb. Der Palast hingegen war nicht mehr bewohnbar. Auch 1.200 der besten Rosse des Herrschers wurden vom "tonner" erschlagen und blieben somit von der Naturkatastrophe nicht verschont. Wie in zahlreichen anderen Türkenschriften auch, geht der Autor auf das Thema Polygamie und Harem kurz ein. Diese trugen maßgeblich zur Ablehnung der Türken bei, da die sogenannte "Vielweiberei" zur "Vorstellung von türkischer Unzucht führte". Aus diesem Grunde waren vor allem Erzählungen über die Harems in Konstantinopel beliebt. "Daraus resultierte die falsche Vorstellung, daß jeder Türke mit einer großen Anzahl von Frauen lebe und, daß alle Türken ausschweifend seien". Göllner hebt letztendlich hervor, dass solche Geschichten gerne kolportiert wurden, "um die Sensationslust der Leser zu befriedigen und den raschen Verkauf der Türkenschriften zu sichern" (Göllner 1979, 312-313).

Am zwölften Tag soll ein großes Erdbeben neben zahreichen schönen Häusern auch die Hälfte des großen Palastes zerstört haben. 2.000 Menschen, dabei 40 "seyner schönsten frawen", sollen dabei umgekommen sein. Istanbul, Edirne, Gelibolu und zahlreiche kleinere Orte hatten mehr als 120.000 Opfer und eine unzählbare Summe von Großvieh zu beklagen. Das angeblich am 12. Juli 1542 stattgefundene Erdbeben soll in Thrakien (Trakya) großen Schaden angerichtet und zahlreiche Opfer gefordert haben. Ambraseys und Finkel heben im Zusammenhang mit dem Erdbeben hervor, dass dieses Ereignis nicht nur in deutschsprachigen, sonden auch in italienischen Schriften thematisiert worden ist. Ambraseys und Finkel, berichten auch von anderen Quellen, die sich mit demselben Erdbeben auseinandersetzen. "In einem auf den 21. November 1542 datierten Brief aus Augsburg wird hervorgehoben, dass infolge des Erdbebens in Istanbul am 20. August 1542, 1700 Häuser eingestürzt und 4500 Menschen gestorben sind" (Ambraseys \& Finkel 2006, 39). Diese Details sind auch in späteren Quellen aufzufinden (Ambraseys \& Finkel 2006, 39). Ambraseys und Finkel heben über derartige Nachrichtenmeldungen hervor, dass das Erscheinen dieser Schriften im europäischen Kontext, meist dann erfolgte, wenn die Beziehungen und die allgemeine politische Lage zum osmanischen Reich durch Instabilität gekennzeichnet war. Auch militärische Erfolge der Osmanen führten dazu, dass Nachrichten dieser Art publiziert wurden, um die westliche Welt zur Gegenwehr zu ermutigen (Ambraseys \& Finkel 2006, 39). Da die osmanischen Quellen keinerlei Hinweise liefern, die diese Nachricht bestätigen könnten, vermutet man, dass diese Nachricht nicht der Realität entspricht. Besonders wichtig sind in diesem Zusammenhang die Quellen des Topkap1 Palastes, in denen ebenfalls diesbezüglich keine Registrierungen aufgefunden werden konnten (Ambraseys \& Finkel, 39). Diese berichten zwar aus dieser Zeit von zahlreichen großen Bränden und Gewittern, aber von großen Erdbebenkatastrophen ist nicht die Rede. Ambraseys und Finkel kommen aufgrunddessen zu der Schlussfolgerung, dass die Erdbebennachrichten infolge von Bränden und Gewittern oder ähnlichen Katastrophen entstanden sind (Ambraseys \& Finkel 2006, 39-40). 
Am 15. Tag nach Mitternacht und zwei Nächte hindurch ist in Konstantinopel ein "Kriegsgeschrey, zu fuß und zu Ro $\beta$ " vernommen worden, welches das Volk in Angst und Schrecken versetzt hat. In derselben Nacht ist außerdem ein Wolfsrudel von 40-50 Wölfen in die Stadt eingebrochen und hat großen Schaden angerichtet. Daraufhin wollte der "gro $\beta$ Türck" am 18. Tag mit einer Schlacht die Stadt vom Wolfsrudel befreien, was ihm aber misslang. Der "groß Türck samt seynen Bassa" beschlossen diesmal bei Sonnenuntergang die Stadt von dieser Plage zu befreien. Nachdem Mustafa Pascha und seine Männer die ganze Nacht hindurch in der Stadt hin und her rennend die Wölfe ohne Erfolg jagten, sprangen diese letztendlich über die Stadtmauer und entfernten sich. Danach soll man von diesen Wölfen weder was gesehen noch gehört haben. Nachrichten über Wölfe wurden vom Leser des 17. Jahrhunderts mit großem Interesse aufgenommen. Diese generell von den Menschen als Katastrophe wahrgenommenen Tiere zählten aufgrunddessen in Literatur und Kunst zu den meist thematisierten Tierarten. Auch wurden sie von den Menschen als bedrohlich und gefährlich eingestuft und galten infolgedessen auch als Sinnbild des Bösen, als das Teuflische auf Erden. Die Wolfskatastrophe wird in der Literatur selten allein, sondern meist mit anderen Katastrophen oder Plagen thematisiert, wie beispielsweise der Heuschrecken- oder Rattenplage oder aber auch den Epidemien (Siemer 2003, 347-348). Die in der Literatur sehr populäre Wolfsfigur ist zum größten Teil Produkt der menschlichen Phantasiekraft. Aber auch in Texten, die auf realen Begebenheiten basieren, wie beispielsweise Nachrichtenmeldungen, ist festzustellen, dass viele phantastische und märchenhafte Elemente miteinfließen. Auch in diesem Text wird die Wolfskatastrophe mit anderen Naturkatastrophen, wie dem Kometen und der Heuschreckenplage zusammen erwähnt. Aus der Perspektive des christlichen Lesers könnte die Vorstellung, dass auch die Türken sich vor Wölfen fürchten, zu einer Art Genugtuung geführt haben. Die Bestrafung der Türken durch derartige Naturkatastrophen, verdeutlicht ja auch eigentlich nichts anderes, als dass dieser furchteinflößende Feind von Gott letztendlich doch auf besondere Art und Weise bestraft wird.

Eingeschüchtert und verunsichert durch diese ungewöhnlichen Ereignisse verlangt der Herrscher des Osmanischen Reiches, die Deutung dieser Geschehnisse. Zwölf weise Männer bzw. im Text auch Astrologen genannt, sollen auslegen, was diese sich täglich ereignenden grausamen Wunderzeichen und Gewitter zu bedeuten haben. Morbech, der älteste unter den Weisen, erklärt dem "König", die Ursache dieser schrecklichen Zeichen. Er hebt hervor, dass dieser "vil Land und Leut verderbet und umgebracht" hat. Zudem erfährt der Leser durch Morbech, dass auch die anderen Astrologen christlichen Glaubens sind. Morbechs Rede gleicht einer Propagandarede, weil er nicht nur die Vorzüge seiner Religion hervorhebt, sondern auch von der Bestrafung des Königs ausgeht, der eine Gefahr für die christliche Religion und die Gläubigen darstellt. Die gesehenen Wunderzeichen hingegen deuten die Astrologen so aus, dass der "König" bald sterben, sein Imperium und damit auch der Islam untergehen wird. Der verärgerte Herrscher möchte seinen Astrologen noch eine letzte Chance geben und teilt ihnen mit, dass sie im Falle einer Bekehrung die bevorstehende Strafe abwenden können. Da die Astrologen aber nicht einwilligen, kommen sie ins Gefängnis. Bald darauf werden sie auf den Marktplatz vor dem Palast des Herrschers gebracht, wo sie alle auf einem Scheiterhaufen verbrannt werden sollen. Plötzlich bricht eine große Finsternis ein, die den Himmel und den Marktplatz über eine Stunde verdeckt, so dass niemand mehr etwas sehen kann. Nach der Finsternis "hat man die zwölff lebendig und unversert, und der ketten entpunden knyend funden". Etliche Paschas bitten nun um das Leben der Weisen. Letztendlich befiehlt der Herrscher auch die Freilassung der Astrologen. Morbech bekommt vom Herrscher sogar eine mit edlen Steinen verzierte Kette geschenkt. Am Ende darf Morbech nach einem geheimen Gespräch mit dem Herrscher, zusammen mit den anderen Astrologen auch zum heiligen Grab. 
Der Herrscher hingegen ist an jenem Tag nicht in der Stadt umhergeritten, was die ganze Stadt und den Hof traurig gestimmt hat. Man vermutete, dass der türkische Herrscher heimlich aus der Stadt geritten sei [Eine andere Nachricht trägt den Titel "Wahrhafftige newe zeitung erschrecklicher dinge, die zu Constantinopel, Handrianopel, Callpoli ... diss vergangen XLII jars in dem Monat Junii sich begeben haben ...” (Weller 1994, 132 - 133; Ebermann 1904, 6061). Auch dieser Nachricht zufolge sollen in Istanbul, Edirne und Gelibolu täglich Wunderzeichen gesehen worden sein. Bis auf einige wenige Details ist diese nahezu mit der obigen Nachricht identisch. Einer dieser Unterschiede ist, dass es während die Astrologen dem Feuer des Scheiterhaufens ausgeliefert waren, nicht zu einer Finsternis kam, sondern das ein Nebel die Stadt verdunkelte. Ein weiterer Unterschied lässt sich am Ende der Geschichte erkennen. Demzufolge flüchtete der türkischer Herrscher entweder heimlich aus der Stadt (Istanbul) als Tausende von Menschen sich zum Christentum bekehrten oder aber er erkrankte vor Angst an einer unheilbaren Krankheit (Weller 1994, 132 - 133; Ebermann 1904, 60-61)].

Die im Text dargestellte große Finsternis wird als weiteres Wunderzeichen gedeutet. Der Text beinhaltet zahlreiche religiöse und märchenhafte Elemente, was an dem feuerspeienden Drachen und dem positiven Ende, m.a.W. der poetischen Gerechtigkeit, zu erkennen ist.

Die Flugschrift repräsentiert nahezu alle Eigenschaften dieser Gattung. Neben genauen Zeitund Ortsangaben spielt auch die Anwesenheit von Zeugen und Zuschauern sowie, die Orte der Öffentlichkeit, wie der Marktplatz, eine wichtige Rolle in den Flugschriften, da sie die Glaubwürdigkeit des Geschehenen erhöhen.

Diese Nachricht soll ihren Lesern die Hoffnung vermitteln, dass die christliche Welt bald von ihrem Glaubensfeind befreit wird. Dass der türkische Herrscher sich von den Wunderzeichen beeinflussen und die Astrologen frei lässt, verweist auf die religiöse und zugleich manipulatorische Funktion dieser Texte. Letztendlich verdeutlicht der Text, dass Nachrichten über Istanbul vom westlichen Leser mit großem Interesse aufgenommen wurden (Faust 1998/1999, Bd. 1, 72). Da in Flugschriften selten Abbildungen vorhanden sind, lassen sich höchstens kleinere Abbildungen, wie beispielsweise die eines Kometen, auffinden.

\section{Schlussfolgerung}

Die politischen und religiösen Auseindersetzungen des 16. Jahrhunderts und die Bedrohung des christlichen Abendlandes durch den türkischen Feind führte zur Publikation zahlreicher Wunderzeichenberichte, in denen die Stadt Istanbul thematisiert wurde.

Am häufigsten wird die Stadt Istanbul und damit verbunden die sogenannte "Türkenfurcht" in den Flugblättern über Himmelserscheinungen ausfindig gemacht. Unter diesen nehmen aber die Kometenflugblätter eindeutig den ersten Rang ein. Die Deutung des Kometen als unheilvolles Zeichen, als Ankündiger von Kriegen und religiösen Konflikten ließ sich aufgrund der permanenten Türkengefahr stets von Neuem instrumentalisieren. Daneben ist die Thematik auch häufig im Bereich der Naturkatastrophenflugblätter, insbesondere auf denen der Erdbebenflugblätter, nachzuweisen [In den Wunderzeichenberichten wird Istanbul am meisten im Zusammenhang mit Himmelszeichen, Naturkatastrophen und seltener mit zoologischen Missgeburten thematisiert]. Die Bestrafung der Türken durch derartige Naturkatastrophen, verdeutlicht ja auch eigentlich nichts anderes, als dass dieser furchteinflößende Feind von Gott letztendlich doch auf besondere Art und Weise bestraft wird. Dass Kometen und Erdbeben dabei den ersten Rang einnehmen, lässt sich unter anderem auch dadurch erklären, dass die Häufigkeit dieser Naturphänomene als Wunderzeichen zugleich auch die geeigneteste war, um auf die Türkenfurcht einzugehen, moralisierend und erschreckend den Leser zu belehren. 
Außerordentliche oder sonderbare Naturphänomene, die als Vorzeichen, als eventuell von Gott zu erwartende Strafen ausgelegt werden, und sich in unmittelbarer Nähe der Menschen abspielen können, lassen darauf hindeuten, dass Gott jederzeit präsent ist und jeder von seiner Strafe getroffen werden kann.

Die Ereignisse in Istanbul beruhen letztlich zum grössten Teil auf realen Geschehnisse, die in abgewandelter und z.T. stark veränderter Form wiedergegeben werden. Meist werden die durch die Naturkatastrophen hervorgerufenen Schäden in übertriebener Weise präsentiert. Zudem macht man auch von Wesen phantastischer Natur, wie beispielsweise Fabelwesen Gebrauch, um somit die Sensationsgier des Lesers anzusprechen. Auch Naturkatastrophen, die sich in keinerlei Weise in der Realität ereignet haben, dienen vermutlich Berichte früherer Ereignisse als Inspirationsquelle, was ein Vergleich mit alten Nachrichtenmeldungen zum Vorschein bringt. 


\section{LITERATURVERZEICHNIS}

Ambraseys N. \& Finkel C. (2006). Türkiye'de ve Komşu Bölgelerde Sismik Etkinlikler. Bir Tarihsel Inceleme, 1500-1800. Çev. Müzeyyen Umur Koçak. Ankara 2006.

Bischoff M. (1986). Himmelszeichen. Eine bildreiche Kunde von Aberglauben und Ängsten. Nördlingen 1986.

Coşan L. (2010). "Funktionen der 'Türckenbüchlei' im 16. Jahrhundert. Dargestellt anhand der Türckenbüchlein von Bernhardin Türck und Johannes Brenz”. Ed. Y. Eğit. Globalisierte Germanistik: Sprache-Literatur-Kultur, XI. Türkischer Internationaler Germanistik Kongress 20.-22. Mai 2009İzmir. İzmir (2010) 169-180.

Cezar M. (1963). “Osmanlı Devrinde İstanbul Yapılarında Tahribat Yapan Yangınlar ve Tabii Âfetler”. 1. Türk San'atı Tarihi Araştırma ve İncelemeleri 1. İstanbul (1963).

Ebermann R. (1904). Die Türkenfurcht, ein Beitrag zur Geschichte der öffentlichen Meinung in Deutschland während der Reformationszeit. Dissertation. Halle a.S.

Ecker, G. (1981). Einblattdrucke von den Anfängen bis 1555. Untersuchungen zu einer Publikationsform literarischer Texte. 2 Bände. Göppingen.

Faust, I. (1998/1999). Zoologische Einblattdrucke und Flugschriften vor 1800. 5 Bände. Stuttgart.

Göllner, C. (1979). Turcica. Die Türkenfrage in der öffentlichen Meinung Europas im 16. Jahrhundert. Bd. 3, 1. Aufl. Baden-Baden: Koerner.

Harms, W. (Hg.) (1985-1997). Deutsche illustrierte Flugblätter des 16. und 17. Jahrhunderts. Bd. I-III: Die Sammlung der Herzog August Bibliothek in Wolfenbüttel, Bd. IV: Die Sammlungen der hessischen Landes- und Hochschulbibliothek in Darmstadt, Bd. VII: Die Sammlung der Zentralbibliothek Zürich, Teil 2: Die Wickiana II (1570-1588). Tübingen.

Harms, W. (1992). "Feindbilder im illustrierten Flugblatt der frühen Neuzeit”. In: Bosbach, F.: Feindbilder. Die Darstellung des Gegners in der politischen Publizistik des Mittelalters und der Neuzeit. Köln, Weimar, Wien: Böhlau.

Heitzer, E. (1995). Das Bild des Kometen in der Kunst: Untersuchungen zur ikonographischen und ikonologischen Tradition des Kometenmotivs in der Kunst vom 14. bis zum 18. Jahrhundert. Gebr. Mann. Berlin.

Hellmann, G. (1921). Die Meteorologie in den deutschen Flugschriften und Flugblättern des XVI. Jahrhunderts. Ein Beitrag zur Geschichte der Meteorologie. Berlin.

Heß, W. (1911). Himmels- und Naturerscheinungen in Einblattdrucken des XV. bis XVIII. Jahrhunderts. Leipzig.

Hofmann, Randall, C. (1999). Monster, Wunder und Kometen. Sensationsberichte auf Flugblättern des 16. bis 18. Jahrhunderts. Eine Ausstellung der Universitätsbibliothek, 19. November - 12. Dezember 1999. (=Schriften der Universitätsbibliothek Erlangen-Nürnberg. Hg. von Hans-Otto Keunecke. Band 36). Erlangen.

Ipşiroğlu, Ş. M. (1980). Meisterwerke islamischer Kunst-Gemälde und Miniaturen im Topkapi-Museum in Istanbul. Stuttgart, Berlin, Köln, Mainz: W. Kohlhammer.

Janeck, A. (1982). Zeichen am Himmel. Flugblätter des 16. Jahrhunderts. 25. Wechselausstellung der Graphischen Sammlung des Germanischen Nationalmuseums Nürnberg 12. März bis 29. August 1982. Hg. vom Germanischen Nationalmuseum Nürnberg, Gerhard Bott. Nürnberg.

Kocadoru, Y. (1990). Die Türken. Studien zu ihrem Bild und seiner Geschichte in Österreich. Dissertation. Klagenfurt.

Köhler, H.-J. (1987). Die Erforschung der Flugschriften des frühen 16. Jahrhunderts als Beitrag zur Presse- und Kommunikationsgeschichte. Ein Situationsbericht zwischen Resignation und Hoffnung. In: Presse und Geschichte II. Neue Beiträge zur historischen Kommunikationsforschung. (=Deutsche Presseforschung. Bd. 26). München.

Langsdorf, K. C. (Hg.) (1819). Des Grafen Laplace philosophischer Versuch über Wahrscheinlichkeiten. Von der dritten Pariser Auflage übersetzt von Friedrich Wilhelm Tönnies. Heidelberg.

Lehmann, H. (1985). Die Kometenflugschriften des 17. Jahrhunderts als historische Quelle. In: Brückner, W. \& Blickle, P. \& Breuer, D. (Hg.): Literatur und Volk im 17. Jahrhundert. Probleme populärer 
Kultur in Deutschland. Teil II. (=Wolfenbütteler Arbeiten zur Barockforschung 13). Wiesbaden (1985) 683-700.

Lindemann, M. (1969). Deutsche Presse bis 1815. Geschichte der deutschen Presse. Teil 1. (=Abhandlungen und Materialien zur Publizistik. Hg. von Fritz Eberhard. Bd. 5. Berlin.

Mauelshagen, F. (1998). Illustrierte Kometenflugblätter in wahrnehmungsgeschichtlicher Perspektive. In: Harms, W.\& Schilling, W. (Hg.): Das illustrierte Flugblatt in der Kultur der Frühen Neuzeit. Wolfenbütteler Arbeitsgespräch 1997. Frankfurt am Main (1998) 101-136.

Rosenberger, V. (1998). Gezähmte Götter. Das Prodigienwesen der römischen Republik. Stuttgart.

Sakin, O. (2002). Tarihsel Kaynaklartyla İstanbul Depremleri. İstanbul: Kitapevi.

Sallmann, M. (2003). 'Innerlichkeit’ und 'Öffentlichkeit' von Religion. Der Fast- und Bettag von 1620 in Basel als offizielle religiöse Bewältigung der Kriegsbedrohung. In: Jakubowski-Tiessen, M. \& Lehmann, H.: Um Himmels Willen: Religion in Katastrophenzeiten. Göttingen: Vandenhoeck\& Ruprecht (2003) 157-178.

Schenda, R. (1963). Die deutschen Prodigiensammlungen des 16. und 17. Jahrhunderts. In: Archiv für Geschichte des Buchwesens. Hg. von der Historischen Kommission des Börsenvereins des Deutschen Buchhandels e.V. Band IV. Frankfurt am Main.

Schenda, R. (1970). Volk ohne Buch. Studien zur Sozialgeschichte der populären Lesestoffe 1770-1910. Frankfurt am Main.

Schilling, M. (1990). Bildpublizistik der frühen Neuzeit. Aufgaben und Leistungen des illustrierten Flugblatts in Deutschland bis um 1700. (=Studien und Texte zur Sozialgeschichte der Literatur. Band 29). Tübingen.

Schwegler, M. (2002). "Erschröckliches Wunderzeichen" oder "natürliches Phänomenon"? Frühneuzeitliche Wunderzeichenberichte aus der Sicht der Wissenschaft. Bayerische Schriften zur Volkskunde, Bd. 7. München.

Schwitalla, J. (1983). Deutsche Flugschriften 1460-1525. Texsortengeschichtliche Studien. Tübingen, Max Niemeyer.

Siemer, S. (2003). "Wölfe in der Stadt. Wahrnehmungsmuster einer Tierkatastrophe am Beispiel des Journal d'un Bourgeois de Paris”. In: Groh, D. \& Kempe, M. \& Mauelshagen, F. (Hg.): Naturkatastrophen: Beiträge zu ihrer Deutung, Wahrnehmung und Darstellung in Text und Bild von der Antike bis ins 20. Jahrhundert. Tübingen: Gunter Narr (2003) 347-365.

Vermij, R. (2003). Erschütterung und Bewältigung. Erdbebenkatastrophen in der frühen Neuzeit. In: Jakubowski-Tiessen, M. \& Lehmann, H.: Um Himmels Willen: Religion in Katastrophenzeiten. Göttingen: Vandenhoeck\& Ruprecht (2003) 235-254.

Weller, E. (1994). Die ersten deutschen Zeitungen: Mit einer Bibliographie (1505-1599).

Zedler, J. H. (1749). Großes vollständiges Universallexikon aller Wissenschaften und Künste. Band 59. Leipzig/ Halle (Stichwörter "Wunder" und "Wunder=Zeichen"). Bd. 59, Sp.2149. (http://www.zedlerlexikon.de/).

Newe zeytung von Constantinopoli. Von eynem Comet/der biß in die 40. tag am hymel ober des Türcken pallast gestanden ist. Von einem fewren Trachen der dem Türcken seinen schatz vnd das new Schloß verprent vnd verderbt hat. Von tonnern/ winden/ hagel vnd schaur/ vnd was schaden sie gethan haben. Von grossen Erdtbidmen. Was zu Constantinopoli Andrinopoli vnd Callipoli geschehen ist. Von den grausamen sachen vn Rumorn von Kriegs geschrey vnd von der grossen suma Wölffen/ so 3. tag in der stat Constantinopoli/ was schaden sie gethan haben. Die auslegung aller obgemelter geschehenen Sachen/Ist geschehen durch zwölff des Türckischen Keysers fürnembste Astronomi/ und sich wie Christen erzeigt haben/ darüb sie der groß Türck hat wöllen verprennen lassen/ un wie sie wunderbarlich bei dem Leben erhalten sein worden/ und wie sie der groß Türck zum heiligen grab verordnet hab. Von der grossen suma Hewschrecken/ die alles das in weytem feld biß in die 20.Welschmeyl verderbt haben (S.I 1542; Res/4 J.germ.36\#Beibd.1 urn:nbn:de:bvb:12-bsb00025126-7 VD16 N 945). http://kruenitz1.unitrier.de. 